\title{
Hilbert Transforms in Clifford Analysis
}

Fred Brackx, Bram De Knock and Hennie De Schepper

\begin{abstract}
The Hilbert transform on the real line has applications in many fields. In particular in one-dimensional signal processing, the Hilbert operator is used to extract global as well as instantaneous characteristics, such as frequency, amplitude and phase, from real signals. The multidimensional approach to the Hilbert transform usually is a tensorial one, considering the so-called Riesz transforms in each of the cartesian variables separately. In this paper we give an overview of generalized Hilbert transforms in Euclidean space, developed within the framework of Clifford analysis. Roughly speaking, this is a function theory of higher dimensional holomorphic functions, which is particularly suited for a treatment of multidimensional phenomena since all dimensions are encompassed at once as an intrinsic feature.
\end{abstract}

\section{Introduction: the Hilbert Transform on the Real Line}

The Hilbert transform is named after D. Hilbert, who, in his studies of integral equations, was the first to observe what is nowadays known as the Hilbert transform pair. However, the Hilbert transform theory was developed mainly by E. C. Titchmarsh and G. H. Hardy. It was Hardy who named it after Hilbert. The Hilbert transform

Fred Brackx

Clifford Research Group, Faculty of Engineering, Ghent University,

Galglaan 2, 9000 Gent, Belgium

e-mail: Freddy.Brackx@UGent.be

Bram De Knock

Clifford Research Group, Faculty of Engineering, Ghent University,

Galglaan 2, 9000 Gent, Belgium

e-mail: bdk@cage.UGent.be

Hennie De Schepper

Clifford Research Group, Faculty of Engineering, Ghent University,

Galglaan 2, 9000 Gent, Belgium

e-mail: Hennie.DeSchepper@UGent.be 
is applied in the theoretical description of many devices and has become an indispensable tool for both global and local descriptions of a signal. It has been directly implemented in the form of Hilbert analogue or digital filters which allow to distinguish different frequency components and therefore locally refine the structure analysis. Those filters are essentially based on the notion of analytic signal, which consists of the linear combination of a bandpass filter, selecting a small part of the spectral information, and its Hilbert transform, the latter basically being the result of a phase shift by $\frac{\pi}{2}$ on the original filter (see e.g. [41]).

For a real one-dimensional finite energy signal $f$, i.e. $f \in L_{2}(\mathbb{R})$, its Hilbert transform on the real line is given by

$$
\mathscr{S}[f](x)=\frac{1}{\pi} \operatorname{Pv} \int_{-\infty}^{+\infty} \frac{f(t)}{x-t} d t,
$$

where $\mathrm{Pv}$ denotes the Cauchy principal value, meaning that in the integral the singularity at $t=x$ is approached in a symmetrical way. Non-finite energy signals, such as (piecewise) constant functions and sines and cosines, should be interpreted as tempered distributions for which the Hilbert transform is defined as the convolution

$$
\mathscr{S}[f](x)=\frac{1}{\pi}\left(\operatorname{Pv} \frac{1}{t} * f(t)\right)(x),
$$

where $\operatorname{Pv} \frac{1}{t}$ is the Principal Value distribution satisfying in distributional sense

$$
\frac{d}{d t} \ln |t|=\operatorname{Pv} \frac{1}{t} \quad \text { and } \quad t \operatorname{Pv} \frac{1}{t}=1 .
$$

In order to recall the fundamental properties of the Hilbert transform on the real line, we introduce the Cauchy integral of a function $f \in L_{2}(\mathbb{R})$ :

$$
\mathscr{C}[f](x, y)=-\frac{1}{2 \pi i} \int_{-\infty}^{+\infty} \frac{f(t)}{(x-t)+i y} d t, \quad y \neq 0 .
$$

This Cauchy integral is, as a function of the complex variable $z=x+i y$, holomorphic in the upper and the lower half of the complex plane and decays to zero for $y \rightarrow \pm \infty$. In other words, for $f \in L_{2}(\mathbb{R})$ its Cauchy integral $\mathscr{C}[f](x, y)$ belongs to the Hardy spaces $H_{2}\left(\mathbb{C}^{ \pm}\right)$, respectively defined by

$$
H_{2}\left(\mathbb{C}^{ \pm}\right)=\left\{F \text { holomorphic in } \mathbb{C}^{ \pm} \text {such that } \sup _{y \gtrless 0} \int_{-\infty}^{+\infty}|F(z)|^{2} d x<+\infty\right\} .
$$

Proposition 1. The Hilbert operator $\mathscr{S}: L_{2}(\mathbb{R}) \rightarrow L_{2}(\mathbb{R})$, (1), enjoys the following properties:

$P(1) \mathscr{S}$ is translation invariant, i.e.

$$
\tau_{a}[\mathscr{S}[f]]=\mathscr{S}\left[\tau_{a}[f]\right],
$$


with $\tau_{a}[f](t)=f(t-a)$.

$P(2) \mathscr{S}$ is dilation invariant, i.e.

$$
d_{a}[\mathscr{S}[f]]=\operatorname{sgn}(a) \mathscr{S}\left[d_{a}[f]\right]
$$

with $d_{a}[f](t)=f(t / a) / \sqrt{|a|}$.

$P(3) \mathscr{S}$ is a linear, bounded and norm-preserving operator.

$P(4) \mathscr{S}$ is invertible with $\mathscr{S}^{-1}=-\mathscr{S}$ and thus $\mathscr{S}^{2}=-\mathbf{1}$.

$P(5) \mathscr{S}$ is unitary, i.e. $\mathscr{S}^{*} \mathscr{S}=\mathscr{S} \mathscr{S}^{*}=\mathbf{1}$.

$P(6) \mathscr{S}$ commutes with differentation, i.e.

$$
\frac{d}{d t}(\mathscr{S}[f](t))=\mathscr{S}\left[\frac{d}{d t} f(t)\right]
$$

$P(7) \mathscr{S}$ arises in a natural way by considering the non-tangential boundary limits (in $L_{2}$ sense) of the Cauchy integral (3), i.e.

$$
\mathscr{C}^{ \pm}[f](x)=\lim _{y \rightarrow 0 \pm} \mathscr{C}[f](x, y)= \pm \frac{1}{2} f(x)+\frac{1}{2} i \mathscr{S}[f](x), \quad x \in \mathbb{R} .
$$

The operators $\mathscr{C}^{ \pm}$are usually called the Cauchy transforms and the formulae (5) in $\mathrm{P}(7)$ are the Plemelj-Sokhotzki formulae in Clifford analysis.

Thus putting $\mathscr{H}=i \mathscr{S}$ we obtain an involutive, norm-preserving, bounded linear operator $\mathscr{H}: L_{2}(\mathbb{R}) \rightarrow L_{2}(\mathbb{R})$, which may be used to define the Hardy space $H_{2}(\mathbb{R})$ as the closed subspace of $L_{2}(\mathbb{R})$ consisting of functions $g$ for which $\mathscr{H}[g]=g$. We call those functions $g \in H_{2}(\mathbb{R})$ analytic signals, inspired by the fact that the nontangential boundary limit $\mathscr{C}^{+}[f]$ of the holomorphic (or analytic) Cauchy integral $\mathscr{C}[f],(3)$, indeed belongs to the Hardy space $H_{2}(\mathbb{R})$. The real and imaginary parts $u=\mathbb{R} e[g]$ and $v=\mathbb{I} m[g]$ of an analytic signal $g$ satisfy the Hilbert formulae

$$
\mathscr{H}[u]=i v \quad \text { and } \quad \mathscr{H}[i v]=u .
$$

It follows that

$$
g=(\mathbf{1}+\mathscr{H})[u] \text { and } g=(\mathbf{1}+\mathscr{H})[i v],
$$

showing that an analytic signal contains redundant information since it can be recovered from its real (or its imaginary) part solely. Note that the Hardy spaces $\mathrm{H}_{2}(\mathbb{R})$ and $\mathrm{H}_{2}\left(\mathbb{C}^{+}\right),(4)$, are isomorphic, since the non-tangential boundary limit for $y \rightarrow 0+$ of $F(z) \in H_{2}\left(\mathbb{C}^{+}\right)$exists a.e. and belongs to $H_{2}(\mathbb{R})$, and the Cauchy integral in $\mathbb{C}^{+}$of $F(x+i 0)$ precisely is $F(z)$.

In frequency space the Hilbert transform, which is convolutional in nature, takes the form of a multiplication operator. Denoting by $\mathscr{F}$ the usual Fourier transform, there holds for a function $f \in L_{2}(\mathbb{R})$ :

$$
\mathscr{F}[\mathscr{H}[f]](\omega)=\operatorname{sgn} \omega \mathscr{F}[f](\omega) \quad \text { and } \quad \mathscr{H}[\mathscr{F}[f]](\omega)=-\mathscr{F}[\operatorname{sgn} t f(t)](\omega) \text {. }
$$


In particular the Fourier spectrum of an analytic signal $g \in H_{2}(\mathbb{R})$ is a causal function with only positive frequencies, and conversely; more explicitly, it reads:

$$
\begin{aligned}
\mathscr{F}[g](\omega) & =\mathscr{F}(1+\mathscr{H})[u](\omega)=(1+\operatorname{sgn} \omega) \mathscr{F}[u](\omega) \\
& =\left\{\begin{array}{cc}
2 \mathscr{F}[u](\omega), & \omega>0 \\
0, & \omega<0
\end{array} .\right.
\end{aligned}
$$

\section{Hilbert Transforms in Euclidean Space}

The Hilbert transform was first generalized to $m$-dimensional Euclidean space by means of the Riesz transforms $R_{j}$ in each of the cartesian co-ordinates $x_{j}, j=$ $1, \ldots, m$, given by

$$
R_{j}[f](\underline{x})=\lim _{\varepsilon \rightarrow 0+0} \frac{2}{a_{m+1}} \int_{\mathbb{R}^{m} \backslash B(\underline{x}, \varepsilon)} \frac{x_{j}-y_{j}}{|\underline{x}-\underline{y}|^{m+1}} f(\underline{y}) d V(\underline{y}),
$$

where $a_{m+1}=\frac{2 \pi^{(m+1) / 2}}{\Gamma((m+1) / 2)}$ denotes the area of the unit sphere $S^{m}$ in $\mathbb{R}^{m+1}$. It was Horváth who, already in his 1953 paper [36], introduced the Clifford vector valued Hilbert operator

$$
\mathscr{S}=\sum_{j=1}^{m} e_{j} R_{j}
$$

The multidimensional Hilbert transform was taken up again in the 1980's and further studied in e.g. $[28,29,35,40,47]$ in the Clifford analysis setting.

Clifford analysis is a function theory which offers an elegant and powerful generalization to higher dimension of the theory of holomorphic functions in the complex plane. In its most simple but still useful setting, flat $m$-dimensional Euclidean space, Clifford analysis focusses on so-called monogenic functions, i.e. null solutions of the Clifford vector valued Dirac operator

$$
\partial_{\underline{x}}=\sum_{j=1}^{m} e_{j} \partial_{x_{j}}
$$

where $\left(e_{1}, \ldots, e_{m}\right)$ forms an orthonormal basis for the quadratic space $\mathbb{R}^{m}$ underlying the construction of the Clifford algebra $\mathbb{R}_{0, m}$, and where the basis vectors satisfy the multiplication rules

$$
e_{j} e_{k}+e_{k} e_{j}=-2 \delta_{j, k}, \quad j, k=1, \ldots, m
$$

Monogenic functions have a special relationship with harmonic functions of several variables: they are refining their properties, since the Dirac operator factorizes the $m$ dimensional Laplacian: $\partial_{\underline{x}}^{2}=-\Delta_{m}$. Euclidean space $\mathbb{R}^{m}$ is embedded in the Clifford algebra $\mathbb{R}_{0, m}$ by identifying the point $\left(x_{1}, \ldots, x_{m}\right) \in \mathbb{R}^{m}$ with the vector variable 


$$
\underline{x}=\sum_{j=1}^{m} e_{j} x_{j}
$$

For more details on Clifford analysis and its applications we refer to e.g. [15,27,30].

\subsection{Definition and properties}

In the framework of Euclidean Clifford analysis, the (Clifford-)Hilbert transform for a suitable function or distribution $f$ is given by

$$
\begin{aligned}
\mathscr{H}[f](\underline{x}) & =\frac{2}{a_{m+1}} \overline{e_{0}} \operatorname{Pv} \int_{\mathbb{R}^{m}} \frac{\underline{\bar{x}}-\bar{y}}{|\underline{x}-\underline{y}|^{m+1}} f(\underline{y}) d V(\underline{y}) \\
& =\frac{2}{a_{m+1}} \overline{e_{0}} \lim _{\varepsilon \rightarrow 0+} \int_{|\underline{x}-\underline{y}|>\varepsilon} \frac{\underline{\bar{x}}-\bar{y}}{|\underline{x}-\underline{y}|^{m+1}} f(\underline{y}) d V(\underline{y}) .
\end{aligned}
$$

In the above expression $e_{0}$ is an additional basis vector for which there also holds

$$
e_{0}^{2}=-1 \quad \text { and } \quad e_{0} e_{j}+e_{j} e_{0}=-2 \delta_{0, j}, j=1, \ldots, m
$$

Furthermore $^{-}$stands for the usual conjugation in the Clifford algebra $\mathbb{R}_{0, m+1}$, i.e. the main anti-involution for which $\overline{e_{j}}=-e_{j}, j=0, \ldots, m$. As in the one-dimensional case there is a strong relationship between the Hilbert transform and the Cauchy integral of a function $f \in L_{2}\left(\mathbb{R}^{m}\right)$. The functions considered here take their values in the Clifford algebra $\mathbb{R}_{0, m+1}$. The space $L_{2}\left(\mathbb{R}^{m}\right)$ is equipped with the $\mathbb{R}_{0, m+1}$ valued inner product and corresponding normsquared:

$$
\langle f, g\rangle=\int_{\mathbb{R}^{m}} \overline{f(\underline{x})} g(\underline{x}) d V(\underline{x}), \quad\|f\|^{2}=[\langle f, f\rangle]_{0},
$$

where $[\lambda]_{0}$ denotes the scalar part of the Clifford number $\lambda$. The Cauchy integral of $f \in L_{2}\left(\mathbb{R}^{m}\right)$ is defined by

$$
\mathscr{C}[f](x)=\mathscr{C}[f]\left(x_{0}, \underline{x}\right)=\frac{1}{a_{m+1}} \int_{\mathbb{R}^{m}} \frac{x_{0}+e_{0}(\underline{x}-\underline{y})}{\left|x_{0}+\underline{x}-\underline{y}\right|^{m+1}} f(\underline{y}) d V(\underline{y}), \quad x_{0} \neq 0 .
$$

Observe the formal similarity with the Cauchy integral (3) of $f \in L_{2}(\mathbb{R}), x_{0}$ taking the rôle of $y$ and the vector $y$ taking the rôle of $t$. It is a (left-)monogenic function in the upper and lower half spaces $\mathbb{R}_{ \pm}^{m+1}=\left\{x_{0} e_{0}+\underline{x}: \underline{x} \in \mathbb{R}^{m}, x_{0} \gtrless 0\right\}$. By a monogenic function in $\mathbb{R}^{m+1}$ is meant a function annihilated by the Cauchy-Riemann operator :

$$
D_{x}=\overline{e_{0}} \partial_{x}=\overline{e_{0}}\left(e_{0} \partial_{x_{0}}+\partial_{\underline{x}}\right)=\partial_{x_{0}}+\overline{e_{0}} \partial_{\underline{x}},
$$

which decomposes the Laplace operator in $\mathbb{R}^{m+1}: D_{x} \overline{D_{x}}=\Delta_{m+1}$. 
Moreover the Cauchy integral decays to zero for $x_{0} \rightarrow \pm \infty$. Summarizing, for a function $f \in L_{2}\left(\mathbb{R}^{m}\right)$, its Cauchy integral $\mathscr{C}[f]\left(x_{0}, \underline{x}\right)$, (18), belongs to the Hardy spaces $H_{2}\left(\mathbb{R}_{ \pm}^{m+1}\right)$, respectively defined by

$$
H_{2}\left(\mathbb{R}_{ \pm}^{m+1}\right)=\left\{D_{x} F=0 \text { in } \mathbb{R}_{ \pm}^{m+1} \text { such that } \sup _{x_{0} \gtrless 0} \int_{-\infty}^{+\infty}\left|F\left(x_{0}+\underline{x}\right)\right|^{2} d x<+\infty\right\} \text {. }
$$

The properties of the multidimensional Hilbert transform are summarized in the following proposition; they show a remarkable similarity with those of the onedimensional Hilbert transform listed in Proposition 1.

Proposition 2. The Hilbert transform $\mathscr{H}: L_{2}\left(\mathbb{R}^{m}\right) \rightarrow L_{2}\left(\mathbb{R}^{m}\right)$ enjoys the following properties:

$P(1) \mathscr{H}$ is translation invariant, i.e.

$$
\tau_{\underline{b}}[\mathscr{H}[f]]=\mathscr{H}\left[\tau_{\underline{b}}[f]\right],
$$

with $\tau_{\underline{b}}[f](\underline{x})=f(\underline{x}-\underline{b}), \underline{b} \in \mathbb{R}^{m}$.

$P(2) \mathscr{H}$ is dilation invariant, i.e.

$$
d_{a}[\mathscr{H}[f]]=\mathscr{H}\left[d_{a}[f]\right],
$$

with $d_{a}[f](\underline{x})=\frac{1}{a^{m / 2}} f(\underline{x} / a), a>0$.

$P(3) \mathscr{H}$ is a norm-preserving, bounded, linear operator.

$P(4) \mathscr{H}$ is an involution and thus invertible with $\mathscr{H}^{-1}=\mathscr{H}$.

$P(5) \mathscr{H}$ is unitary with $\mathscr{H}^{*}=\mathscr{H}$.

$P(6) \mathscr{H}$ anticommutes with the Dirac operator (12).

$P(7) \mathscr{H}$ arises in a natural way by considering the non-tangential boundary limits in $L_{2}$ sense of the Cauchy integral (18):

$$
\mathscr{C}^{ \pm}[f](\underline{x})=\lim _{x_{0} \rightarrow 0 \pm} \mathscr{C}[f]\left(x_{0}, \underline{x}\right)= \pm \frac{1}{2} f(\underline{x})+\frac{1}{2} \mathscr{H}[f](\underline{x}), \quad \underline{x} \in \mathbb{R}^{m} .
$$

In distributional sense this boundary behaviour is explicited by

$$
E(0 \pm, \underline{x})=\lim _{x_{0} \rightarrow 0 \pm} E\left(x_{0}, \underline{x}\right)= \pm \frac{1}{2} \delta(\underline{x})+\frac{1}{2} \mathscr{K}(\underline{x}),
$$

where $E\left(x_{0}, \underline{x}\right)$ is the fundamental solution of the Cauchy-Riemann operator $D_{x}$, (19):

$$
D_{x} E\left(x_{0}, \underline{x}\right)=D_{x}\left(\frac{1}{a_{m+1}} \frac{x_{0}-\overline{e_{0}} \underline{x}}{\left|x_{0}+e_{0} \underline{x}\right|^{m+1}}\right)=\delta\left(x_{0}, \underline{x}\right),
$$

and $\mathscr{K}$ is the Hilbert convolution kernel:

$$
\mathscr{H}[f]=\mathscr{K} * f=\frac{2}{a_{m+1}} \overline{e_{0}} \operatorname{Pv} \frac{\bar{x}}{|\underline{x}|^{m+1}} * f .
$$


As each function in the Hardy space $H_{2}\left(\mathbb{R}_{ \pm}^{m+1}\right),(20)$, possesses a non-tangential $L_{2}$ boundary limit for $x_{0} \rightarrow 0 \pm$, one is lead to the introduction of the Hardy space $H_{2}\left(\mathbb{R}^{m}\right)$ as the closure in $L_{2}\left(\mathbb{R}^{m}\right)$ of the non-tangential boundary limits $F(\underline{x}+0)$ for $x_{0} \rightarrow 0+$ of the functions $F\left(x_{0}, \underline{x}\right)$ in $H_{2}\left(\mathbb{R}_{+}^{m+1}\right)$. As moreover the Cauchy integral of $F(\underline{x}+0)$ is precisely $F\left(x_{0}, \underline{x}\right)$, we may conclude that the Hardy spaces $H_{2}\left(\mathbb{R}_{+}^{m+1}\right)$ and $H_{2}\left(\mathbb{R}^{m}\right)$ are isomorphic.

As the Hardy space $H_{2}\left(\mathbb{R}^{m}\right)$ is, by definition, a closed subspace of the space of $L_{2}\left(\mathbb{R}^{m}\right)$, the latter space may be decomposed as the orthogonal direct sum

$$
L_{2}\left(\mathbb{R}^{m}\right)=H_{2}\left(\mathbb{R}^{m}\right) \oplus H_{2}\left(\mathbb{R}^{m}\right)^{\perp} .
$$

The corresponding projection operators are precisely the Cauchy transforms $\pm \mathscr{C}^{ \pm}$ since it can be directly verified that

$$
\begin{aligned}
& f=\mathscr{C}^{+}[f]-\mathscr{C}^{-}[f] \\
& \mathscr{C}^{+}\left[\mathscr{C}^{+}[f]\right]=\mathscr{C}^{+}[f] \\
& \left(-\mathscr{C}^{-}\right)\left[-\mathscr{C}^{-}[f]\right]=\left(-\mathscr{C}^{-}\right)[f] \\
& \mathscr{C}^{+}\left[\mathscr{C}^{-}[f]\right]=\mathscr{C}^{-}\left[\mathscr{C}^{+}[f]\right]=0 \\
& \left\langle\mathscr{C}^{+}[f], \mathscr{C}^{-}[f]\right\rangle_{L_{2}}=0
\end{aligned}
$$

The analytic signal $\mathscr{C}^{+}[f] \in H_{2}\left(\mathbb{R}^{m}\right)$ and the anti-analytic signal $\left(-\mathscr{C}^{-}[f]\right) \in$ $H_{2}\left(\mathbb{R}^{m}\right)^{\perp}$ thus possess a monogenic extension to $H_{2}\left(\mathbb{R}_{ \pm}^{m+1}\right)$ respectively. Note that the Hardy space $H_{2}\left(\mathbb{R}^{m}\right)$ and its orthogonal complement $H_{2}\left(\mathbb{R}^{m}\right)^{\perp}$ are nicely characterized by means of the Hilbert and Cauchy transforms:

Lemma 1. A function $g \in L_{2}\left(\mathbb{R}^{m}\right)$ belongs to $H_{2}\left(\mathbb{R}^{m}\right)$ if and only if $\mathscr{H}[g]=g$, or $\mathscr{C}^{+}[g]=g$, or $\mathscr{C}^{-}[g]=0$.

Lemma 2. A function $h \in L_{2}\left(\mathbb{R}^{m}\right)$ belongs to $H_{2}\left(\mathbb{R}^{m}\right)^{\perp}$ if and only if $\mathscr{H}[g]=-g$, or $\mathscr{C}^{+}[h]=0$, or $\mathscr{C}^{-}[h]=-h$.

\subsection{Analytic signals}

Because of the properties mentioned in the preceding subsection, the functions in $H_{2}\left(\mathbb{R}^{m}\right)$ already deserve to be called analytic signals in $\mathbb{R}^{m}$. But then their frequency contents should show a property similar to one-dimensional causality (9), thus involving a multidimensional analogue of the Heaviside step function. As in the one-dimensional case the Hilbert transform (15) in frequency space takes the form of a multiplication operator; for a function $f \in L_{2}\left(\mathbb{R}^{m}\right)$ there holds:

$$
\mathscr{F}[\mathscr{H}[f]](\underline{y})=\overline{e_{0}} i \underline{\xi} \mathscr{F}[f](\underline{y}) \quad \text { and } \quad \mathscr{H}[\mathscr{F}[f]](\underline{y})=e_{0} \mathscr{F}[i \underline{\omega} f(\underline{x})](\underline{y}),
$$

where $\mathscr{F}$ denotes the standard Fourier transform in $\mathbb{R}^{m}$ given by 


$$
\mathscr{F}[f(\underline{x})](\underline{y})=\int_{\mathbb{R}^{m}} f(\underline{x}) \exp (-i\langle\underline{x}, \underline{y}\rangle) d V(\underline{x}),
$$

and $\underline{\omega}=\underline{x} /|\underline{x}|$ and $\xi=y /|y|$ may be interpreted as the multidimensional analogues of the signum-function $\operatorname{sgn}(x)=x /|x|$ on the real line. As an aside these formulae allow the practical computation of the Hilbert transform by means of the Fourier transform:

$$
\mathscr{H}[f](\underline{x})=\mathscr{F}^{-1}\left[\overline{e_{0}} i \underline{\xi} \mathscr{F}[f](\underline{y})\right] .
$$

The Fourier spectrum of the Cauchy transforms $\mathscr{C}^{ \pm}[f]$ (21) of a function $f \in$ $L_{2}\left(\mathbb{R}^{m}\right)$ then read

$$
\begin{aligned}
\mathscr{F}\left[\mathscr{C}^{ \pm}[f]\right] & = \pm \frac{1}{2} \mathscr{F}[f]+\frac{1}{2} \overline{e_{0}} i \underline{\xi} \mathscr{F}[f] \\
& = \pm \psi_{ \pm} \mathscr{F}[f]
\end{aligned}
$$

where we have introduced the mutually annihilating idempotents

$$
\psi_{+}=\frac{1}{2}\left(1+\overline{e_{0}} i \underline{\omega}\right) \quad \text { and } \quad \psi_{-}=\frac{1}{2}\left(1-\overline{e_{0}} i \underline{\omega}\right),
$$

satisfying the following properties:
(i) $\psi_{ \pm}^{2}=\psi_{ \pm}$;
(ii) $\psi_{+} \psi_{-}=\psi_{-} \psi_{+}=0$;
(iii) $\psi_{+}+\psi_{-}=1$;
(iv) $\psi_{+}-\psi_{-}=\overline{e_{0}} i \underline{\omega}$;
(v) $i e_{0} \underline{\omega} \psi_{ \pm}= \pm \psi_{ \pm}$.

The functions $\psi_{ \pm},(30)$, thus are the Clifford algebra valued multidimensional analogues to the Heaviside step function. They were introduced independently by Sommen [46] and McIntosh [40]. They allow for the practical computation of the Cauchy transforms of a function $f \in L_{2}\left(\mathbb{R}^{m}\right)$ through

$$
\mathscr{C}^{ \pm}[f]=\mathscr{F}^{-1}\left[ \pm \psi_{ \pm} \mathscr{F}[f]\right]
$$

which will be used in the next subsection. Now take an analytic signal $g \in H_{2}\left(\mathbb{R}^{m}\right)$, then, in accordance with Lemma $1, g=\mathscr{H}[g]$ or $g=\frac{1}{2}(g+\mathscr{H}[g])=\mathscr{C}^{+}[g]$ and $\mathscr{C}^{-}[g]=0$ from which it follows that

$$
\mathscr{F}[g]=\mathscr{F}\left[\mathscr{C}^{+}[g]\right]=\psi_{+} \mathscr{F}[g],
$$

whereas, trivially,

$$
\mathscr{F}\left[\mathscr{C}^{-}[g]\right]=-\psi_{-} \mathscr{F}[g]=0,
$$

which is the multidimensional counterpart to the "vanishing negative frequencies" in one dimension. 
We now show that, similarly to the splitting of a complex signal into its real and imaginary parts, see (6), a Clifford algebra valued analytic signal can be split into two components satisfying multidimensional Hilbert formulae. To that end we observe that, by the introduction of the additional basis vector $e_{0}$, the Clifford algebra $\mathbb{R}_{0, m+1}$ may be decomposed, using two copies of the Clifford algebra $\mathbb{R}_{0, m}$, as follows:

$$
\mathbb{R}_{0, m+1}=\mathbb{R}_{0, m} \oplus \overline{e_{0}} \mathbb{R}_{0, m} .
$$

Thus if $g$ is a $\mathbb{R}_{0, m+1}$ valued analytic signal, it can be written as $g=u+\overline{e_{0}} v$, where $u$ and $v$ are $\mathbb{R}_{0, m}$ valued functions satisfying, in view of Lemma 1 ,

$$
\mathscr{H}[u]=\overline{e_{0}} v \text { and } \mathscr{H}\left[\overline{e_{0}} v\right]=u .
$$

This means that an analytic signal $g$ is completely determined by one of its components $u$ or $v$ :

$$
g=(\mathbf{1}+\mathscr{H})[u]=(\mathbf{1}+\mathscr{H})\left[\overline{e_{0}} v\right],
$$

and moreover shows a Fourier spectrum only containing $\psi_{+}$-frequencies and doubling those of $u$ or $v$ :

$$
\mathscr{F}[g]=\left(1+\overline{e_{0}} i \underline{\xi}\right) \mathscr{F}[u]=\left(1+\overline{e_{0}} i \underline{\xi}\right) \mathscr{F}\left[\overline{e_{0}} v\right]=2 \psi_{+} \mathscr{F}[u]=2 \psi_{+} \mathscr{F}\left[\overline{e_{0}} v\right] .
$$

Similar considerations hold for anti-analytic signals in $H_{2}\left(\mathbb{R}^{m}\right)^{\perp}$.

\subsection{Monogenic extensions of analytic signals}

For any $f \in L_{2}\left(\mathbb{R}^{m}\right)$ the Cauchy transforms $\pm \mathscr{C}^{ \pm}[f],(21)$, are (anti-)analytic signals, thus showing monogenic extensions to $\mathbb{R}_{ \pm}^{m}$. A first possibility to construct these monogenic extensions is by using the Cauchy integral, leading to the monogenic functions

$$
\begin{array}{r}
\mathscr{C}\left[\mathscr{C}^{+}[f]\right]= \begin{cases}\mathscr{C}[f] & \text { in } \mathbb{R}_{+}^{m} \\
0 & \text { in } \mathbb{R}_{-}^{m}\end{cases} \\
\mathscr{C}\left[-\mathscr{C}^{-}[f]\right]= \begin{cases}0 & \text { in } \mathbb{R}_{+}^{m} \\
-\mathscr{C}[f] & \text { in } \mathbb{R}_{-}^{m}\end{cases}
\end{array}
$$

which moreover tend to zero for $x_{0} \rightarrow \pm \infty$. However there is also another way to construct monogenic extensions to $\mathbb{R}^{m+1}$ of functions in $\mathbb{R}^{m}$, albeit that they have to be real-analytic. This method, the so-called Cauchy-Kowalewskaia extension principle, is a typical construct of Clifford analyis; for a given real-analytic function $\phi$ in $\mathbb{R}^{m}$, a monogenic extension in an open neighbourhood in $\mathbb{R}^{m+1}$ of $\mathbb{R}^{m}$ is given by

$$
C K[\phi]=\exp \left(-x_{0} \overline{e_{0}} \partial_{\underline{x}}\right)[\phi]=\sum_{j=0}^{+\infty} \frac{(-1)^{j}}{j !} x_{0}^{j}\left(\overline{e_{0}} \partial_{\underline{x}}\right)^{j}[\phi] .
$$


In particular the scalar valued, and real-analytic, Fourier kernel $\exp (i\langle\underline{x}, \underline{y}\rangle)$ in $\mathbb{R}^{m}$ is monogenically extended to the whole of $\mathbb{R}^{m+1}$ by

$$
C K[\exp (i\langle\underline{x}, \underline{y}\rangle)]=\sum_{j=0}^{+\infty} \frac{(-1)^{j}}{j !} x_{0}^{j}\left(\overline{e_{0}} i \underline{y}\right)^{j}[\exp (i\langle\underline{x}, \underline{y}\rangle)]=\exp \left(-i x_{0} \overline{e_{0}} \underline{y}\right) \exp (i\langle\underline{x}, \underline{y}\rangle),
$$

which takes its values in $\operatorname{span}_{\mathbb{C}}\left(\overline{\overline{e_{0}}} e_{1}, \ldots, \overline{\overline{e_{0}}} e_{m}\right)$.

In view of (31), i.e.

$$
\mathscr{C}^{+}[f]=\mathscr{F}^{-1}\left[\psi_{+} \mathscr{F}[f]\right],
$$

we thus obtain, following an idea of [43] and [38], as a monogenic extension of $\mathscr{C}^{+}[f]$ :

$$
C K\left[\mathscr{C}^{+}[f]\right]\left(x_{0}, \underline{x}\right)=(2 \pi)^{-m} \int_{\mathbb{R}^{m}} \exp (i\langle\underline{x}, \underline{y}\rangle) \exp \left(-i x_{0} \overline{\overline{e_{0}}} \underline{y}\right) \psi_{+} \mathscr{F}[f](\underline{y}) d V(\underline{y}) .
$$

A direct computation yields

$$
\begin{aligned}
C K & {\left[\mathscr{C}^{+}[f]\right]\left(x_{0}, \underline{x}\right) } \\
& =(2 \pi)^{-m} \int_{\mathbb{R}^{m}} \exp (i\langle\underline{x}, \underline{y}\rangle) \exp \left(-x_{0} \rho\right) \psi_{+} \mathscr{F}[f](\underline{y}) d V(\underline{y}) \\
& =(2 \pi)^{-m} \int_{S^{m-1}} \psi_{+} d S(\underline{\xi}) \int_{0}^{+\infty} \exp \left(\left(i\langle\underline{x}, \underline{\xi}\rangle-x_{0}\right) \rho\right) \rho^{m-1} \mathscr{F}[f](\rho \underline{\xi}) d \rho,
\end{aligned}
$$

since

$$
\exp \left(-i x_{0} \overline{e_{0}} \underline{y}\right) \psi_{+}=\exp \left(-x_{0} \rho\right) \psi_{+},
$$

where we have once more used spherical co-ordinates with $\underline{y}=\rho \underline{\xi}$. This further leads to

$$
C K\left[\mathscr{C}^{+}[f]\right]\left(x_{0}, \underline{x}\right)=(2 \pi)^{-m} \int_{S^{m-1}} \psi_{+} d S(\underline{\xi}) \mathscr{L}\left[\rho^{m-1} \mathscr{F}[f](\rho \underline{\xi})\right]\left(x_{0}-i\langle\underline{x}, \underline{\xi}\rangle\right),
$$

where $\mathscr{L}$ denotes the Laplace transform. It is clear that this monogenic extension tends to zero only when $x_{0} \rightarrow+\infty$. Thus, with restriction to $\mathbb{R}_{+}^{m}$ we obtain:

$$
\mathscr{C}[f]\left(x_{0}, \underline{x}\right)=(2 \pi)^{-m} \int_{S^{m-1}} \psi_{+} d S(\underline{\xi}) \mathscr{L}\left[\rho^{m-1} \mathscr{F}[f](\rho \underline{\xi})\right]\left(x_{0}-i\langle\underline{x}, \underline{\xi}\rangle\right), x_{0}>0 .
$$

In a similar way we obtain in $\mathbb{R}_{-}^{m}$ :

$$
\mathscr{C}[f]\left(x_{0}, \underline{x}\right)=(2 \pi)^{-m} \int_{S^{m-1}} \psi_{-} d S(\underline{\xi}) \mathscr{L}\left[\rho^{m-1} \mathscr{F}[f](\rho \underline{\xi})\right]\left(-x_{0}-i\langle\underline{x}, \underline{\xi}\rangle\right), x_{0}<0 .
$$




\subsection{Example 1}

The direct sum decomposition of finite energy signals goes through for tempered distributions and even more so for compactly supported distributions. Let us illustrate this by the case of the delta- or Dirac-distribution $\delta(\underline{x})$ in $\mathbb{R}^{m}$. Its Cauchy integral is given by

$$
\mathscr{C}[\delta]\left(x_{0}, \underline{x}\right)=E\left(x_{0}, \underline{x}\right) * \delta(\underline{x})=E\left(x_{0}, \underline{x}\right)=\frac{1}{a_{m+1}} \frac{x_{0}-\overline{e_{0}} \underline{x}}{\left|x_{0} e_{0}+\underline{x}\right|^{m+1}},
$$

which is monogenic in $\mathbb{R}_{ \pm}^{m+1}$ and even in $\mathbb{R}^{m+1} \backslash\{0\}$ w.r.t. the Cauchy-Riemann operator $D_{x}(19)$. This implies that as long as $\underline{x} \neq 0$ there is a continuous transition of this Cauchy integral over $\mathbb{R}^{m}$ as the common boundary of $\mathbb{R}_{+}^{m+1}$ and $\mathbb{R}_{-}^{m+1}$. Thus the "jump" over $\mathbb{R}^{m}$ of $\mathscr{C}[\delta]\left(x_{0}, \underline{x}\right)$ will occur at $\underline{x}=0$, and indeed

$$
\mathscr{C}^{ \pm}[\delta](\underline{x})= \pm \frac{1}{2} \delta(\underline{x})+\frac{1}{2} \mathscr{K}(\underline{x}),
$$

with $\mathscr{K}$ the Hilbert kernel (24), since

$$
\mathscr{H}[\delta](\underline{x})=\mathscr{K} * \delta(\underline{x})=\mathscr{K}(\underline{x})=\frac{2}{a_{m+1}} \overline{e_{0}} \operatorname{Pv} \frac{\underline{\bar{x}}}{|\underline{x}|^{m+1}} .
$$

The direct sum decomposition of the Dirac-distribution $\delta(\underline{x})$ now follows readily:

$$
\delta(\underline{x})=\left(\frac{1}{2} \delta(\underline{x})+\frac{1}{2} \mathscr{K}(\underline{x})\right)+\left(\frac{1}{2} \delta(\underline{x})-\frac{1}{2} \mathscr{K}(\underline{x})\right) .
$$

The Cauchy integral of the first component is given by

$$
\mathscr{C}\left[\mathscr{C}^{+}[\delta]\right]= \begin{cases}\mathscr{C}[\delta]=E\left(x_{0}, \underline{x}\right) & \text { in } \mathbb{R}_{+}^{m+1} \\ 0 & \text { in } \mathbb{R}_{-}^{m+1}\end{cases}
$$

while the Cauchy integral of the second component is given by

$$
\mathscr{C}\left[-\mathscr{C}^{-}[\delta]\right]=\left\{\begin{array}{ll}
0 & \text { in } \mathbb{R}_{+}^{m+1} \\
-\mathscr{C}[\boldsymbol{\delta}]=-E\left(x_{0}, \underline{x}\right) & \text { in } \mathbb{R}_{-}^{m+1}
\end{array} .\right.
$$

As the Hilbert transform is involutive we obtain for the transform of the Hilbert kernel itself:

$$
\mathscr{H}[\mathscr{K}](\underline{x})=\mathscr{H}^{2}[\delta](\underline{x})=\delta(\underline{x}),
$$

which is confirmed by the convolution

$$
\mathscr{H}[\mathscr{K}]=\mathscr{K} * \mathscr{K}=\frac{4}{a_{m+1}^{2}} \operatorname{Pv} \frac{\bar{x}}{|\underline{x}|^{m+1}} * \operatorname{Pv} \frac{\bar{x}}{|\underline{x}|^{m+1}}=\delta(\underline{x}) .
$$

This leads to the direct sum decomposition of the Hilbert kernel $\mathscr{K}(\underline{x})$ : 


$$
\mathscr{K}(\underline{x})=\left(\frac{1}{2} \mathscr{K}(\underline{x})+\frac{1}{2} \delta(\underline{x})\right)+\left(\frac{1}{2} \mathscr{K}(\underline{x})-\frac{1}{2} \delta(\underline{x})\right)
$$

where both components may be monogenically extended through their Cauchy integral to respectively $\mathbb{R}_{ \pm}^{m+1}$ by the functions $\pm E\left(x_{0}, \underline{x}\right)$. Note in this connection that $\left( \pm \mathscr{C}^{ \pm}\right)[\boldsymbol{\delta}]=\mathscr{C}^{ \pm}[\mathscr{K}]$.

As the delta-distribution $\delta(\underline{x})$ is $\mathbb{R}_{0, m}$ valued — in fact real valued - and its Hilbert transform $\mathscr{K}(\underline{x})$ is $\overline{e_{0}} \mathbb{R}_{0, m}$ valued, they sum up to an $\mathbb{R}_{0, m+1}$ valued analytic signal $\delta(\underline{x})+\mathscr{K}(\underline{x})$ which has its frequencies supported by $\psi_{+}$and doubling those of $\delta(\underline{x})$. This is confirmed by the following results in frequency space. For the standard Fourier transform (27) we have $\mathscr{F}[\delta]=1$ and thus

$$
\mathscr{F}[\mathscr{K}]=\mathscr{F}\left[\frac{2}{a_{m+1}} \operatorname{Pv} \frac{\bar{x}}{|\underline{x}|^{m+1}}\right]=\overline{e_{0}} i \underline{\xi}
$$

and thus also

$$
\mathscr{F}[\delta(\underline{x})+\mathscr{K}(\underline{x})]=1+\overline{e_{0}} i \underline{\xi}=2 \psi_{+} .
$$

As already mentioned the (anti-)analytic signals $\pm \mathscr{C}^{ \pm}[\delta](\underline{x})=\frac{1}{2} \delta(\underline{x}) \pm \frac{1}{2} \mathscr{K}(\underline{x})=$ $\mathscr{C}^{ \pm}[\mathscr{K}](\underline{x})$ may be monogenically extended to $\mathbb{R}_{ \pm}^{m+1}$ by the functions $\pm E\left(x_{0}, \underline{x}\right) \in$ $H_{2}\left(\mathbb{R}_{ \pm}^{m+1}\right)$ respectively, defined in (23). Alternatively the Cauchy-Kowalewskaia technique (40) leads to

$$
C K\left[\mathscr{C}^{+}[\delta]\right]=(2 \pi)^{-m} \int_{S^{m-1}} \psi_{+} d S(\underline{\xi}) \mathscr{L}\left[\rho^{m-1}\right]\left(x_{0}-i\langle\underline{x}, \underline{\xi}\rangle\right), x_{0}>0
$$

and

$$
C K\left[-\mathscr{C}^{-}[\delta]\right]=(2 \pi)^{-m} \int_{S^{m-1}} \psi_{-} d S(\underline{\xi}) \mathscr{L}\left[\rho^{m-1}\right]\left(-x_{0}-i\langle\underline{x}, \underline{\xi}\rangle\right), x_{0}<0
$$

As $\mathscr{L}\left[\rho^{m-1}\right]=\frac{\Gamma(m)}{z^{m}}$ for $\mathbb{R} e(z)>0$ we arrive at

$$
C K\left[\mathscr{C}^{+}[\delta]\right]=\frac{(m-1) !}{(2 \pi)^{m}} \int_{S^{m-1}} \frac{\psi_{+}}{\left(x_{0}-i\langle\underline{x}, \underline{\xi}\rangle\right)^{m}} d S(\underline{\xi}), x_{0}>0
$$

and

$$
C K\left[-\mathscr{C}^{-}[\delta]\right]=\frac{(m-1) !}{(2 \pi)^{m}} \int_{S^{m-1}} \frac{\psi_{-}}{\left(-x_{0}-i\langle\underline{x}, \underline{\xi}\rangle\right)^{m}} d S(\underline{\xi}), x_{0}<0 .
$$

But $i \psi_{+}=-\overline{e_{0}} \underline{\xi} \psi_{+}$and $i \psi_{-}=\overline{e_{0}} \underline{\xi} \psi_{-}$from which it follows that

$$
\frac{\psi_{+}}{\left(x_{0}-i\langle\underline{x}, \underline{\xi}\rangle\right)^{m}}=\frac{\psi_{+}}{\left(x_{0}+\langle\underline{x}, \underline{\xi}\rangle \overline{e_{0}} \underline{\xi}\right)^{m}}
$$

and 


$$
\frac{\psi_{-}}{\left(x_{0}+i\langle\underline{x}, \underline{\xi}\rangle\right)^{m}}=\frac{\psi_{-}}{\left(x_{0}+\langle\underline{x}, \underline{\xi}\rangle \overline{e_{0}} \underline{\xi}\right)^{m}} .
$$

Moreover the $C K$-extensions under consideration $C K\left[\mathscr{C}^{+}[\delta]\right]=E\left(x_{0}, \underline{x}\right), x_{0}>0$ and $C K\left[-\mathscr{C}^{-}[\delta]\right]=-E\left(x_{0}, \underline{x}\right), x_{0}<0$ both are $\mathbb{R}_{0, m+1}$ valued, so their compleximaginary parts should vanish, which implies that

$$
\int_{S^{m-1}} \frac{\underline{\xi}}{\left(x_{0}+\langle\underline{x}, \underline{\xi}\rangle \overline{e_{0}} \underline{\xi}\right)^{m}} d S(\underline{\xi})=0,
$$

finally leading to

$$
E\left(x_{0}, \underline{x}\right)=\frac{1}{2} \frac{(m-1) !}{(2 \pi)^{m}} \int_{S^{m-1}} \frac{1}{\left(x_{0}+\langle\underline{x}, \underline{\xi}\rangle \overline{e_{0}} \underline{\xi}\right)^{m}} d S(\underline{\xi}), x_{0}>0
$$

and

$$
E\left(x_{0}, \underline{x}\right)=\frac{(-1)^{m-1}}{2} \frac{(m-1) !}{(2 \pi)^{m}} \int_{S^{m-1}} \frac{1}{\left(x_{0}+\langle\underline{x}, \underline{\xi}\rangle \overline{e_{0}} \underline{\xi}\right)^{m}} d S(\underline{\xi}), x_{0}<0 .
$$

\subsection{Example 2}

Again we start with a scalar valued tempered distribution

$$
u(\underline{x})=\exp (i\langle\underline{a}, \underline{x}\rangle)=\cos \langle\underline{a}, \underline{x}\rangle+i \sin \langle\underline{a}, \underline{x}\rangle,
$$

with $\underline{a}$ a non-zero constant Clifford vector, for which we put $\underline{\alpha}=\underline{a} /|\underline{a}|$.

From one-dimensional theory it is known that the Hilbert transform $\mathscr{H}=i \mathscr{S}$ acts as a rotator, mapping $\cos a x$ and $\sin a x$ on $i \operatorname{sgn}(a) \sin a x$ and $-i \operatorname{sgn}(a) \cos a x$ respectively. It is now shown that the Clifford-Hilbert transform (15) enjoys a similar property in higher dimension. We have successively

$$
\mathscr{F}[u(\underline{x})](\underline{y})=(2 \pi)^{m} \delta(\underline{y}-\underline{a}),
$$

and thus

$\mathscr{F}[\mathscr{H}[u(\underline{x})]](\underline{y})=\overline{e_{0}} i \underline{\xi} \mathscr{F}[u(\underline{x})](\underline{y})=(2 \pi)^{m} \overline{e_{0}} i \underline{\xi} \delta(\underline{y}-\underline{a})=(2 \pi)^{m} i \overline{e_{0}} \underline{\alpha} \delta(\underline{y}-\underline{a})$,

from which it follows that

$$
\mathscr{H}[u](\underline{x})=i \overline{e_{0}} \underline{\alpha} \exp (i\langle\underline{a}, \underline{x}\rangle)=\overline{e_{0}} \underline{\alpha}(-\sin \langle\underline{a}, \underline{x}\rangle+i \cos \langle\underline{a}, \underline{x}\rangle),
$$

and thus 


$$
\mathscr{H}[\cos \langle\underline{a}, \underline{x}\rangle]=-\left(\overline{e_{0}} \underline{\alpha}\right) \sin \langle\underline{a}, \underline{x}\rangle
$$

and

$$
\mathscr{H}[\sin \langle\underline{a}, \underline{x}\rangle]=\left(\overline{e_{0}} \underline{\alpha}\right) \cos \langle\underline{a}, \underline{x}\rangle .
$$

Note that $\underline{\alpha}=\underline{a} /|\underline{a}|$ is the multidimensional counterpart to the one-dimensional $\operatorname{sgn}(a)$ and that $\left(\overline{e_{0}} \underline{\alpha}\right)^{2}=-1$.

We also obtain the following analytic signals:

(i) $\cos \langle\underline{a}, \underline{x}\rangle-\left(\overline{e_{0}} \underline{\alpha}\right) \sin \langle\underline{a}, \underline{x}\rangle=\exp \left(-\left(\overline{e_{0}} \underline{\alpha}\right)\langle\underline{a}, \underline{x}\rangle\right)$;

(ii) $\sin \langle\underline{a}, \underline{x}\rangle+\left(\overline{e_{0}} \underline{\alpha}\right) \cos \langle\underline{a}, \underline{x}\rangle=\left(\overline{e_{0}} \underline{\alpha}\right) \exp \left(-\left(\overline{e_{0}} \underline{\alpha}\right)\langle\underline{a}, \underline{x}\rangle\right)$;

(iii) $\left(1+i\left(\overline{e_{0}} \underline{\alpha}\right)\right) \exp (i\langle\underline{a}, \underline{x}\rangle)=\left(1+i\left(\overline{e_{0}} \underline{\alpha}\right)\right) \exp \left(-\left(\overline{e_{0}} \underline{\alpha}\right)\langle\underline{a}, \underline{x}\rangle\right)$.

\section{Generalized Hilbert Transforms in Euclidean space}

In the early 2000's, four broad families $T_{\lambda, p}, U_{\lambda, p}, V_{\lambda, p}$ and $W_{\lambda, p}$, with $\lambda \in \mathbb{C}$ and $p \in \mathbb{N}_{0}$, of specific distributions in Clifford analysis were introduced and studied by Brackx, Delanghe and Sommen (see $[16,17]$ ) and it was shown that the Hilbert kernel $\mathscr{K}$, introduced in the preceding section, is one of those distributions acting as a convolution operator (see e.g. [18]). Later on, those distributions were normalized and thoroughly discussed in a series of papers [4-6, 8-10,14]. We recall the definitions of those normalized distributions, where $l \in \mathbb{N}_{0}$ :

$$
\begin{aligned}
& \left\{\begin{array}{l}
T_{\lambda, p}^{*}=\pi^{\frac{\lambda+m}{2}+p} \frac{T_{\lambda, p}}{\Gamma\left(\frac{\lambda+m}{2}+p\right)}, \quad \lambda \neq-m-2 p-2 l \\
T_{-m-2 p-2 l, p}^{*}=\frac{(-1)^{p} l ! \pi^{\frac{m}{2}-l}}{2^{2 p+2 l}(p+l) ! \Gamma\left(\frac{m}{2}+p+l\right)} P_{p}(\underline{x}) \partial_{\underline{x}}^{2 p+2 l} \delta(\underline{x}),
\end{array}\right. \\
& \left\{\begin{array}{l}
U_{\lambda, p}^{*}=\pi^{\frac{\lambda+m+1}{2}+p} \frac{U_{\lambda, p}}{\Gamma\left(\frac{\lambda+m+1}{2}+p\right)}, \quad \lambda \neq-m-2 p-2 l-1 \\
U_{-m-2 p-2 l-1, p}^{*}=\frac{(-1)^{p+1} l ! \pi^{\frac{m}{2}-l}}{2^{2 p+2 l+1}(p+l) ! \Gamma\left(\frac{m}{2}+p+l+1\right)}\left(\partial_{\underline{x}}^{2 p+2 l+1} \delta(\underline{x})\right) P_{p}(\underline{x})
\end{array}\right. \\
& \left\{\begin{array}{l}
V_{\lambda, p}^{*}=\pi^{\frac{\lambda+m+1}{2}+p} \frac{V_{\lambda, p}}{\Gamma\left(\frac{\lambda+m+1}{2}+p\right)}, \quad \lambda \neq-m-2 p-2 l-1 \\
V_{-m-2 p-2 l-1, p}^{*}=\frac{(-1)^{p+1} l ! \pi^{\frac{m}{2}-l}}{2^{2 p+2 l+1}(p+l) ! \Gamma\left(\frac{m}{2}+p+l+1\right)} P_{p}(\underline{x})\left(\partial_{\underline{x}}{ }^{2 p+2 l+1} \delta(\underline{x})\right)
\end{array}\right.
\end{aligned}
$$




$$
\left\{\begin{array}{l}
W_{\lambda, p}^{*}=\pi^{\frac{\lambda+m}{2}+p} \frac{W_{\lambda, p}}{\Gamma\left(\frac{\lambda+m}{2}+p\right)}, \quad \lambda \neq-m-2 p-2 l \\
W_{-m-2 p-2 l, p}^{*}=\frac{(-1)^{p+1} l ! \pi^{\frac{m}{2}-l}}{2^{2 p+2 l+2}(p+l+1) ! \Gamma\left(\frac{m}{2}+p+l+1\right)} \underline{x} P_{p}(\underline{x}) \underline{x} \partial_{\underline{x}}^{2 p+2 l+2} \delta(\underline{x})
\end{array}\right.
$$

the action of the original distributions $T_{\lambda, p}, U_{\lambda, p}, V_{\lambda, p}$ and $W_{\lambda, p}$ on a testing function $\phi$ being given by

$$
\begin{aligned}
\left\langle T_{\lambda, p}, \phi\right\rangle & =a_{m}\left\langle F p r_{+}^{\mu+p_{e}}, \Sigma_{p}^{(0)}[\phi]\right\rangle \\
\left\langle U_{\lambda, p}, \phi\right\rangle & =a_{m}\left\langle F p r_{+}^{\mu+p_{e}}, \Sigma_{p}^{(1)}[\phi]\right\rangle \\
\left\langle V_{\lambda, p}, \phi\right\rangle & =a_{m}\left\langle F p r_{+}^{\mu+p_{e}}, \Sigma_{p}^{(3)}[\phi]\right\rangle \\
\left\langle W_{\lambda, p}, \phi\right\rangle & =a_{m}\left\langle F p r_{+}^{\mu+p_{e}}, \Sigma_{p}^{(2)}[\phi]\right\rangle
\end{aligned}
$$

We explain the notations in the above expressions. First, the symbol $F p$ stands for the well-known distribution "finite parts" on the real line, furthermore $\mu=\lambda+m-1$ and $p_{e}$ denotes the "even part of $p$ ", defined by $p_{e}=p$ if $p$ is even and $p_{e}=p-1$ if $p$ is odd. Finally, $\Sigma_{p}^{(0)}, \Sigma_{p}^{(1)}, \Sigma_{p}^{(2)}$ and $\Sigma_{p}^{(3)}$ are the generalized spherical mean operators defined on scalar valued testing functions $\phi$ by

$$
\begin{aligned}
& \Sigma_{p}^{(0)}[\phi]=r^{p-p_{e}} \Sigma^{(0)}\left[P_{p}(\underline{\omega}) \phi(\underline{x})\right]=\frac{r^{p-p_{e}}}{a_{m}} \int_{S^{m-1}} P_{p}(\underline{\omega}) \phi(\underline{x}) d S(\underline{\omega}) \\
& \Sigma_{p}^{(1)}[\phi]=r^{p-p_{e}} \Sigma^{(0)}\left[\underline{\omega} P_{p}(\underline{\omega}) \phi(\underline{x})\right]=\frac{r^{p-p_{e}}}{a_{m}} \int_{S^{m-1}} \underline{\omega} P_{p}(\underline{\omega}) \phi(\underline{x}) d S(\underline{\omega}) \\
& \Sigma_{p}^{(2)}[\phi]=r^{p-p_{e}} \Sigma^{(0)}\left[\underline{\omega} P_{p}(\underline{\omega}) \underline{\omega} \phi(\underline{x})\right]=\frac{r^{p-p_{e}}}{a_{m}} \int_{S^{m-1}} \underline{\omega} P_{p}(\underline{\omega}) \underline{\omega} \phi(\underline{x}) d S(\underline{\omega})(85) \\
& \Sigma_{p}^{(3)}[\phi]=r^{p-p_{e}} \Sigma^{(0)}\left[P_{p}(\underline{\omega}) \underline{\omega} \phi(\underline{x})\right]=\frac{r^{p-p_{e}}}{a_{m}} \int_{S^{m-1}} P_{p}(\underline{\omega}) \underline{\omega} \phi(\underline{x}) d S(\underline{\omega})
\end{aligned}
$$

where $P_{p}(\underline{\omega})$ is an inner spherical monogenic of degree $p$, i.e. a restriction to the unit sphere $S^{m-1}$ of a monogenic homogeneous polynomial in $\mathbb{R}^{m}$.

Making use of those Clifford distributions, we have then constructed two possible generalizations of the Hilbert transform (15), aiming at preserving as much as possible of its traditional properties $\mathrm{P}(1)-\mathrm{P}(7)$ listed in Proposition 2 (see also $[5,8])$. It is shown that in each case some of the properties - different ones - are inevitably lost. Nevertheless we will obtain in Section 3.1 a bounded singular integral operator on $L_{2}\left(\mathbb{R}^{m}\right)$ and in Section 3.2 a bounded singular integral operator on appropriate Sobolev spaces. 


\subsection{First generalization}

In the first approach the Hilbert transform is generalized by using other kernels for the convolution, stemming from the families of distributions mentioned above. They constitute a refinement of the generalized Hilbert kernels introduced by Horváth in [37], who considered convolution kernels, homogeneous of degree $(-m)$, of the form

$$
P v \frac{S(\underline{\omega})}{r^{m}}, \quad \underline{x}=r \underline{\omega}, \quad r=|\underline{x}|, \quad \underline{\omega} \in S^{m-1},
$$

where $S(\underline{\omega})$ is a surface spherical harmonic. We investigate generalized Hilbert convolution kernels which are homogeneous of degree $(-m)$ as well, however involving inner and outer spherical monogenics. We already have mentioned that an inner spherical monogenic is the restriction to the unit sphere $S^{m-1}$ of a monogenic homogeneous polynomial in $\mathbb{R}^{m}$. An outer spherical monogenic is the restriction to the unit sphere $S^{m-1}$ of a monogenic homogeneous function in the complement of the origin; an example of an outer spherical monogenic is the "signum" function $\underline{\omega}$ since it is the restriction to $S^{m-1}$ of the function $\underline{x} /|\underline{x}|^{m+1}$ which is monogenic in $\mathbb{R}^{m} \backslash\{\underline{0}\}$.

We consider the following specific distributions

$$
\begin{aligned}
T_{-m-p, p} & =F p \frac{1}{r^{m}} P_{p}(\underline{\omega})=P v \frac{P_{p}(\underline{\omega})}{r^{m}} \\
U_{-m-p, p} & =F p \frac{1}{r^{m}} \underline{\omega} P_{p}(\underline{\omega})=P v \frac{\underline{\omega} P_{p}(\underline{\omega})}{r^{m}} \\
V_{-m-p, p} & =F p \frac{1}{r^{m}} P_{p}(\underline{\omega}) \underline{\omega}=P v \frac{P_{p}(\underline{\omega}) \underline{\omega}}{r^{m}} \\
W_{-m-p, p} & =F p \frac{1}{r^{m}} \underline{\omega} P_{p}(\underline{\omega}) \underline{\omega}=P v \frac{\underline{\omega} P_{p}(\underline{\omega}) \underline{\omega}}{r^{m}} \\
P v \frac{S_{p+1}(\underline{\omega})}{r^{m}} & =-\frac{1}{2(p+1)}\left(U_{-m-p, p}+V_{-m-p, p}\right) \\
P v \frac{\omega}{\frac{\omega}{S_{p+1}(\underline{\omega})}} & =-\frac{1}{r^{m}}\left(W_{-m-p, p}-T_{-m-p, p}\right)
\end{aligned}
$$

where $P_{p}(\underline{x})=\partial_{\underline{x}} S_{p+1}(\underline{x}), S_{p+1}(\underline{x})$ being a scalar valued solid spherical harmonic and hence $P_{p}(\underline{x})$ being a vector valued solid spherical monogenic. These distributions are homogeneous of degree $(-m)$ and the functions occurring in the numerator satisfy the cancellation condition

$$
\int_{S^{m-1}} \Omega(\underline{\omega}) d \underline{\omega}=0,
$$

$\Omega(\underline{\omega})$ being either of $P_{p}(\underline{\omega}), \underline{\omega} P_{p}(\underline{\omega}), P_{p}(\underline{\omega}) \underline{\omega}$ or $\underline{\omega} P_{p}(\underline{\omega}) \underline{\omega}$.

Their Fourier symbols, given by (see [10]) 


$$
\begin{aligned}
& \mathscr{F}\left[T_{-m-p}\right]=i^{-p} \pi^{\frac{m}{2}} \frac{\Gamma\left(\frac{p}{2}\right)}{\Gamma\left(\frac{m+p}{2}\right)} P_{p}(\underline{\omega}) \\
& \mathscr{F}\left[U_{-m-p}\right]=i^{-p-1} \pi^{\frac{m}{2}} \frac{\Gamma\left(\frac{p+1}{2}\right)}{\Gamma\left(\frac{m+p+1}{2}\right)} \underline{\omega} P_{p}(\underline{\omega}) \\
& \mathscr{F}\left[V_{-m-p}\right]=i^{-p-1} \pi^{\frac{m}{2}} \frac{\Gamma\left(\frac{p+1}{2}\right)}{\Gamma\left(\frac{m+p+1}{2}\right)} P_{p}(\underline{\omega}) \underline{\omega} \\
& \mathscr{F}\left[W_{-m-p}\right]=i^{-p-2} \pi^{\frac{m}{2}} \frac{p \Gamma\left(\frac{p}{2}\right)}{(m+p) \Gamma\left(\frac{m+p}{2}\right)}\left(\underline{\omega} P_{p}(\underline{\omega}) \underline{\omega}-\frac{m-2}{p} P_{p}(\underline{\omega})\right)
\end{aligned}
$$

are homogeneous of degree 0 and moreover are bounded functions, whence

$$
T_{-m-p, p} * f, \quad U_{-m-p, p} * f, \quad V_{-m-p, p} * f, \quad W_{-m-p, p} * f
$$

are bounded Singular Integral Operators on $L_{2}\left(\mathbb{R}^{m}\right)$ which are direct generalizations of the Hilbert transform $\mathscr{H}(15)$, preserving (properly adapted analogues of the) properties $\mathrm{P}(1)-\mathrm{P}(3)$.

We now investigate whether the new operators (94) will fulfil some appropriate analogues of the remaining properties $\mathrm{P}(4)-\mathrm{P}(7)$ as well. To this end we closely examine the kernel $T_{-m-p, p}$. First we observe that

$$
T_{-m-p, p} * T_{-m-p, p}=\frac{(-1)^{p}}{2^{p}} \pi^{\frac{m}{2}} \frac{\Gamma\left(\frac{m}{2}\right)}{\Gamma(p)}\left[\frac{\Gamma\left(\frac{p}{2}\right)}{\Gamma\left(\frac{m+p}{2}\right)}\right]^{2} T_{-m, p} P_{p}\left(\partial_{\underline{x}}\right),
$$

which directly implies that the generalized Hilbert transform $T_{-m-p, p} * f$ does not satisfy an analogue of property $\mathrm{P}(4)$. Next, as it can easily be shown that the considered operator coincides with its adjoint - up to a minus sign when $p$ is even we may also conclude, in view of (95), that it will not be unitary, neither does it commute with the Dirac operator.

Finally, the most important drawback of this first generalization is undoubtedly the fact that we cannot establish an analogue of property $\mathrm{P}(7)$, since it turned out impossible to find a generalized Cauchy kernel in $\mathbb{R}^{m+1} \backslash\{0\}$, for which a part of the boundary values is precisely the generalized Hilbert kernel $T_{-m-p, p}$. Similar conclusions hold for the other generalized kernels used in (88). 


\subsection{Second generalization}

Subsequent to the observations made in the previous subsection, we now want to find a type of generalized Hilbert kernel which actually preserves property $\mathrm{P}(7)$. To that end, we define the function

$E_{p}(x)=E_{p}\left(x_{0}, \underline{x}\right)=\frac{1}{a_{m+1, p}} \frac{\bar{x} e_{0}}{|x|^{m+1+2 p}} P_{p}(\underline{x})=\frac{1}{a_{m+1, p}} \frac{x_{0}+e_{0} \underline{x}}{\left|x_{0} e_{0}+\underline{x}\right|^{m+1+2 p}} P_{p}(\underline{x})$,

where

$$
a_{m+1, p}=\frac{(-1)^{p}}{2^{p}} \frac{2 \pi^{\frac{m+1}{2}}}{\Gamma\left(\frac{m+1}{2}+p\right)}
$$

involving a homogeneous polynomial $P_{p}(\underline{x})$ of degree $p$ which we take once more to be vector valued and monogenic. It is stated in the next proposition that these functions $E_{p}$ are good candidates for generalized Cauchy kernels. Note that for $p=1$ and taking $P_{0}(\underline{x})=1$, the standard Cauchy kernel, i.e. the fundamental solution of the Cauchy-Riemann operator $D_{x}$ in $\mathbb{R}^{m+1}$ is reobtained.

For the proofs of all results mentioned in the remainder of this section, we refer the reader to [8].

Proposition 3. The function $E_{p}$, (96), satisfies the following properties:

(i) $E_{p} \in L_{1}^{\text {loc }}\left(\mathbb{R}^{m+1}\right)$ and $\lim _{|x| \rightarrow \infty} E_{p}(x)=0, \forall p \in \mathbb{N}$;

(ii) $D_{x} E_{p}(x)=P_{p}\left(\partial_{\underline{x}}\right) \delta(x)$ in distributional sense, $\forall p \in \mathbb{N}$;

Here, $L_{1}^{\text {loc }}\left(\mathbb{R}^{m+1}\right)$ stands for the locally integrable functions on $\mathbb{R}^{m+1}$.

Next we calculate their non-tangential distributional boundary values for $x_{0} \rightarrow$ $0 \pm$. To that end we first formulate an interesting auxiliary result in the following lemma.

Lemma 3. For $p \in \mathbb{N}_{0}$ one has

$$
\lim _{x_{0} \rightarrow 0+} \frac{x_{0}}{\left|x_{0}+\underline{x}\right|^{m+1+2 p}}=\frac{1}{2^{p+1} p !} a_{m+1, p} \partial_{\underline{x}}^{2 p} \delta(\underline{x}) .
$$

$a_{m+1, p}$ being given by (97).

Proposition 4. For each $p \in \mathbb{N}_{0}$ one has

$$
\begin{aligned}
& E_{p}(0+, \underline{x})=\lim _{x_{0} \rightarrow 0+} E_{p}\left(x_{0}, \underline{x}\right)=\frac{1}{2} P_{p}\left(\partial_{\underline{x}}\right) \delta(\underline{x})+\frac{1}{2} \mathscr{K}_{p}(\underline{x}), \\
& E_{p}(0-, \underline{x})=\lim _{x_{0} \rightarrow 0-} E_{p}\left(x_{0}, \underline{x}\right)=-\frac{1}{2} P_{p}\left(\partial_{\underline{x}}\right) \delta(\underline{x})+\frac{1}{2} \mathscr{K}_{p}(\underline{x}),
\end{aligned}
$$

where 


$$
\mathscr{K}_{p}(\underline{x})=\frac{2}{a_{m+1, p}} \overline{e_{0}} F p \frac{\underline{\bar{\omega}} P_{p}(\underline{\omega})}{r^{m+p}}=-\frac{2}{a_{m+1, p}} \overline{e_{0}} U_{-m-2 p, p}^{*} .
$$

The distribution $\mathscr{K}_{p}$ (101) arising in the previous proposition allows for the definition of a generalized Hilbert transform $\mathscr{H}_{p}$, given by

$$
\mathscr{H}_{p}[f]=\mathscr{K}_{p} * f .
$$

Because the Fourier symbol

$$
\mathscr{F}\left[\mathscr{K}_{p}\right]=-\frac{2}{a_{m+1, p}} \overline{e_{0}} i^{-p-1} U_{0, p}^{*}
$$

of the kernel $\mathscr{K}_{p}$ is not a bounded function, the operator $\mathscr{H}_{p}$, (102), will also not be bounded on $L_{2}\left(\mathbb{R}^{m}\right)$. However, the Fourier symbol (103) is polynomial of degree $p$, implying that $\mathscr{H}_{p}$ is a bounded operator between the Sobolev spaces $W_{2}^{n}\left(\mathbb{R}^{m}\right) \rightarrow$ $W_{2}^{n-p}\left(\mathbb{R}^{m}\right)$, for $n \geq p$. It can indeed be proved that

Proposition 5. The generalized Cauchy integral $\mathscr{C}_{p}$, given by $\mathscr{C}_{p}[f]=E_{p} * f$, maps the Sobolev space $W_{2}^{n}\left(\mathbb{R}^{m}\right)$ into the Hardy space $H^{2}\left(\mathbb{R}_{+}^{m+1}\right)$, for each natural number $n \geq p$.

Corollary 1. The generalized Hilbert transform $\mathscr{H}_{p},(102)$, is a bounded linear operator between the Sobolev spaces $W_{2}^{n}\left(\mathbb{R}^{m}\right)$ and $W_{2}^{n-p}\left(\mathbb{R}^{m}\right)$, for each natural number $n \geq p$.

Comparing further the properties of $\mathscr{H}_{p}$ with those of the standard Hilbert transform $\mathscr{H}$ in Clifford analysis shows that the main objective for this second generalization is fulfilled on account of Proposition 4: the kernel $\mathscr{K}_{p}$ arises as a part of the boundary values of a generalized Cauchy kernel $E_{p}$, which constitutes an analogue of the "classical" property $\mathrm{P}(7)$. However, the kernel $\mathscr{K}_{p}$ is a homogeneous distribution of degree $(-m-p)$, meaning that $\mathscr{H}_{p}$ is not dilation invariant.

\section{The Anisotropic Hilbert Transform}

The (generalized) multidimensional Hilbert transforms on $\mathbb{R}^{m}$ considered in Sections 2 and 3 might be characterized as isotropic, since the metric in the underlying space is the standard Euclidean one. In this section we adopt the idea of an anisotropic (also called metric dependent or metrodynamical) Clifford setting, which offers the possibility of adjusting the co-ordinate system to preferential, not necessarily mutually orthogonal, directions intrinsically present in the $m$ dimensional structures or signals to be analyzed. In this new area of Clifford analysis (see e.g. [22,31]), we have constructed the so-called anisotropic (Clifford-)Hilbert transform (see [7,11]), a special case of which was already introduced and used for two-dimensional image processing in [34]. 


\subsection{Definition of the anisotropic Hilbert transform}

For the basic language of anisotropic Clifford analysis, we first present the notion of metric tensor, namely a real, symmetric and positive definite tensor $\widetilde{G}=$ $\left(g_{k l}\right)_{k, l=0, \ldots, m}$ of order $(m+1)$ which gives rise to two bases in $\mathbb{R}^{m+1}$ : a covariant basis $\left(e_{0}, \ldots, e_{m}\right)$ and a contravariant basis $\left(e^{0}, \ldots, e^{m}\right)$ corresponding to each other through the metric tensor, viz

$$
e_{k}=\sum_{l=0}^{m} g_{k l} e^{l} \quad \text { and } \quad e^{l}=\sum_{k=0}^{m} g^{l k} e_{k}, \quad \text { with } \quad \widetilde{G}^{-1}=\left(g^{k l}\right)_{k, l=0, \ldots, m}
$$

Then, a Clifford algebra is constructed, depending on the metric tensor involved, and all necessary definitions and results of Euclidean Clifford analysis are adapted to this metric dependent setting. We mention e.g. that the classical scalar product is replaced by the symmetric bilinear form

$$
\langle x, y\rangle_{\widetilde{G}}=\sum_{k=0}^{m} \sum_{l=0}^{m} g_{k l} x^{k} y^{l} .
$$

The anisotropic Dirac and Cauchy-Riemann operators in $\mathbb{R}^{m+1}$ take the form

$$
\partial_{\widetilde{G}}=\sum_{k=0}^{m} e^{k} \partial_{x^{k}}
$$

and

$$
D_{\widetilde{G}}=\bar{e}_{0} \partial_{\widetilde{G}}=\partial_{x^{0}}+\bar{e}_{0} \underline{\partial}_{G} .
$$

where $G=\left(g_{k l}\right)_{k, l=1, \ldots, m}$ in $\mathbb{R}^{m \times m}$ is the subtensor of the metric tensor $\widetilde{G}$ in $\mathbb{R}^{(m+1) \times(m+1)}$. The fundamental solution of the latter operator,

$$
E_{\widetilde{G}}(x)=\frac{1}{a_{m+1}} \frac{\bar{x} e^{0}}{\left(\langle x, x\rangle_{\widetilde{G}}\right)^{(m+1) / 2}},
$$

is now used as the kernel in the definition of the metrodynamical Cauchy integral given, for a function $f \in L_{2}\left(\mathbb{R}^{m}\right)$ or a tempered distribution, by

$$
\mathscr{C}_{\widetilde{G}}[f]=E_{\widetilde{G}} * f,
$$

which is monogenic in $\mathbb{R}_{+}^{m+1}$ (and in $\mathbb{R}_{-}^{m+1}$ ). Taking limits in $L_{2}$ or distributional sense for $x^{0} \rightarrow 0+$ gives, through careful calculation (see [11]):

$$
\lim _{x^{0} \rightarrow 0+} \mathscr{C}_{\widetilde{G}}[f]=\frac{1}{\sqrt{\operatorname{det} \widetilde{G}}}\left(\frac{1}{2} f+\frac{1}{2} \bar{e}^{0} H_{\text {ani }} * f\right),
$$

with 


$$
H_{a n i}(\underline{x})=\sqrt{\operatorname{det} \widetilde{G}}\left(\frac{2}{a_{m+1}} \operatorname{Pv} \frac{\underline{\underline{x}}}{\left(\langle\underline{x}, \underline{x}\rangle_{G}\right)^{(m+1) / 2}}\right),
$$

Similarly, for $x^{0} \rightarrow 0-$, we obtain

$$
\lim _{x^{0 \rightarrow 0-}} \mathscr{C}_{\widetilde{G}}[f]=\frac{1}{\sqrt{\operatorname{det} \widetilde{G}}}\left(-\frac{1}{2} f+\frac{1}{2} \bar{e}^{0} H_{a n i} * f\right) .
$$

The above results are the anisotropic Plemelj-Sokhotski formulae and they give rise to the definition of the anisotropic Hilbert transform:

$$
\mathscr{H}_{\text {ani }}[f]=\bar{e}^{0} H_{\text {ani }} * f .
$$

As already mentioned in the introduction of this section, for $m=2$, such an anisotropic Hilbert transform was considered in [34], however for the special case where the $e_{0}$-direction in $\mathbb{R}^{3}$ is chosen perpendicular to the $\mathbb{R}^{2}$-plane spanned by $\left(e_{1}, e_{2}\right)$. This corresponds to a $\widetilde{G}$-matrix of order 3 in which $g_{01}=g_{02}=0$.

\subsection{Properties of the anisotropic Hilbert transform}

In order to study the properties of the linear operator $\mathscr{H}_{\text {ani }}$, (113), we will also have to pass to frequency space, so we need to introduce a proper definition for the anisotropic Fourier transform on $\mathbb{R}^{m}$ in the present metric dependent setting:

$$
\mathscr{F}_{G}[f](\underline{x})=\int_{\mathbb{R}^{m}} \exp \left(-2 \pi i\langle\underline{x}, \underline{y}\rangle_{G}\right) f(\underline{y}) d V(\underline{y})=\int_{\mathbb{R}^{m}} \exp \left(-2 \pi i \underline{x}^{T} G \underline{y}\right) f(\underline{y}) d V(\underline{y}) .
$$

Due to the assumed symmetric character of the tensor $G$ it is found that

$$
\mathscr{F}_{G}[f](\underline{x})=\mathscr{F}[f](G \underline{x}) .
$$

The following properties of $\mathscr{H}_{\text {ani }}$ may then be proved (see [11]):

(P1) $\mathscr{H}_{\text {ani }}$ is translation invariant.

(P2) $\mathscr{H}_{a n i}$ is dilation invariant, which is equivalent to its kernel $H_{\text {ani }}$, (111), being a homogeneous distribution of degree $-m$.

(P3) $\mathscr{H}_{\text {ani }}$ is a bounded operator on $L_{2}\left(\mathbb{R}^{m}\right)$, which is equivalent to its Fourier symbol

$$
\mathscr{F}_{G}\left[H_{\text {ani }}\right](\underline{x})=\sqrt{\frac{\operatorname{det} \widetilde{G}}{\operatorname{det} G}} i \frac{\underline{x}}{\langle\underline{x}, \underline{x}\rangle_{G}}
$$

being a bounded function.

(P4) Up to a metric related constant, $\mathscr{H}_{\text {ani }}$ squares to unity, more explicitly

$$
\left(\mathscr{H}_{\text {ani }}\right)^{2}=\frac{\operatorname{det} \widetilde{G}}{\operatorname{det} G} \mathbf{1} .
$$


(P5) $\mathscr{H}_{\text {ani }}$ is selfadjoint.

(P6) $\mathscr{H}_{\text {ani }}$ arises in a natural way by considering non-tangential boundary values of the Cauchy integral $\mathscr{C}_{\widetilde{G}}$, (109), in $\mathbb{R}^{m+1}$.

Note that the anisotropic Hilbert transform shows the influence of the underlying metric in two different ways: (1) the determinant of the "mother" metric $\widetilde{G}$ on $\mathbb{R}^{m+1}$ arises as an explicit factor in the expression for the kernel, and (2) the induced metric $G$ on $\mathbb{R}^{m}$ comes into play explicitly through the denominator of the kernel, but also implicitly through its numerator since the vector $\underline{x}$ contains the (skew) basis vectors $\left(e_{k}\right)_{k=1}^{m}$.

The particularity of this metric dependence may also be seen in frequency space, where the metric $G$ not only arises in the Fourier symbol (116) of $\mathscr{H}_{\text {ani }}$, but is also hidden in the definition of the anisotropic Fourier transform itself, while the "mother" metric $\widetilde{G}$ again only is seen to arise through its determinant.

The above observations do raise the question whether there exists a one-to-one correspondence between a given Hilbert transform on $\left(\mathbb{R}^{m}, G\right)$ and the associated Cauchy integral on $\left(\mathbb{R}^{m+1}, \widetilde{G}\right)$ from which it originates, or in other words: does the Hilbert transform contain enough geometrical information to completely determine the "mother" metric $\widetilde{G}$ ? The answer is negative. It turns out that, given a Hilbert kernel

$$
H_{a n i}=c\left(\frac{2}{a_{m+1}} \operatorname{Pv} \frac{\underline{\bar{x}}}{(\langle\underline{x}, \underline{x}\rangle)^{(m+1) / 2}}\right),
$$

being dependent on the $m$-dimensional metric $G$ and on the strictly positive constant $c$, it is part of the boundary value of a Cauchy kernel in $\left(\mathbb{R}^{m+1}, \widetilde{G}\right)$, with

$$
\widetilde{G}=\left(\begin{array}{cc}
g_{00} & \underline{u}^{T} \\
\underline{u} & G
\end{array}\right),
$$

where $\left(g_{00}, \underline{u}^{T}\right)$ are characterized, but not uniquely determined, by the equation

$$
g_{00}-\underline{u}^{T} G^{-1} \underline{u}=\frac{c}{\operatorname{det} G} .
$$

\subsection{Example}

It is interesting to demonstrate the difference between the Clifford-Hilbert transform of Section 2 and its anisotropic counterpart. So consider in $\mathbb{R}^{m}$ again the scalar valued tempered distribution $f(\underline{x})=\exp (i\langle\underline{a}, \underline{x}\rangle)$, where $\underline{a}$ is a constant, non-zero Clifford vector.

In the isotropic case we found (see Section 2.4) 


$$
\mathscr{H}[f](\underline{x})=i \overline{e_{0}} \frac{\underline{a}}{|\underline{a}|} \exp (i\langle\underline{a}, \underline{x}\rangle) .
$$

In the anisotropic case we successively obtain

$$
\mathscr{F}_{G}[f](\underline{y})=\mathscr{F}[f](G \underline{y})=\delta(G \underline{y}-\underline{a}),
$$

and thus

$$
\mathscr{F}_{G}\left[\mathscr{H}_{G, c}[f]\right](\underline{y})=\overline{e^{0}} i \sqrt{\frac{\operatorname{det}(\widetilde{G})}{\operatorname{det}(G)}} \frac{G^{-1} \underline{a}}{\left|G^{-1} \underline{a}\right|_{G}} \delta(G \underline{y}-\underline{a}),
$$

with

$$
\left|G^{-1} \underline{a}\right|_{G}=\left[\left(G^{-1} \underline{a}\right)^{T} G\left(G^{-1} \underline{a}\right)\right]^{\frac{1}{2}}=\left[\underline{a}^{T} G^{-1} \underline{a}\right]^{\frac{1}{2}} .
$$

Subsequent calculations reveal that

$$
\begin{aligned}
& \mathscr{F}_{G}^{-1}[\delta(G \underline{y}-\underline{a})](\underline{x})=\int_{\mathbb{R}^{m}} \exp \left(i \underline{x}^{T} G \underline{y}\right) \delta(G \underline{y}-\underline{a}) d V(\underline{y}) \\
& =\frac{1}{\operatorname{det}(G)} \int_{\mathbb{R}^{m}} \exp \left(i \underline{x}^{T} \underline{y}^{\prime}\right) \delta\left(\underline{y}^{\prime}-\underline{a}\right) d V\left(\underline{y}^{\prime}\right)=\frac{1}{\operatorname{det}(G)} \exp (i\langle\underline{a}, \underline{x}\rangle)
\end{aligned}
$$

Hence

$$
\mathscr{H}_{\text {ani }}[f](\underline{x})=i \overline{e^{0}} \sqrt{\frac{\operatorname{det}(\widetilde{G})}{(\operatorname{det}(G))^{3}}} \frac{G^{-1} \underline{a}}{\left|G^{-1} \underline{a}\right|} \exp (i\langle\underline{a}, \underline{x}\rangle) .
$$

\section{Conclusion}

The concept of analytic signal on the real time-axis is fundamental in signal processing. It contains the original signal as well as its Hilbert transform, and allows for the decomposition of a finite energy signal into its analytic and anti-analytic components. In mathematical terms this is rephrased as the direct sum decomposition of $L_{2}(\mathbb{R})$ into the Hardy space $H_{2}(\mathbb{R})$ and its orthogonal complement, and the analytic signals are precisely the functions in $H_{2}(\mathbb{R})$. In this paper we have presented several generalizations of the Hilbert transform and the corresponding analytic signal to Euclidean space of arbitrary dimension and we have indicated the properties which are characteristic in the one-dimensional case and persist in each of those generalizations. It becomes apparent, also from the given examples, that the Clifford analysis framework is most appropriate to develop these multidimensional Hilbert transforms. That Clifford analysis could be a powerful tool in multidimensional signal analysis became already clear during the last decade from the several constructions of multidimensional Fourier transforms with quaternionic or Clifford algebra valued kernels with direct applications in signal analysis and pattern recognition, see $[20,21,24,32-34,39]$ and also the review paper [23] wherein the relations between the different approaches are established. In view of the fact that in the underly- 
ing paper the interaction of the Clifford-Hilbert transforms with only the standard Fourier transform was considered, their interplay with the various Clifford-Fourier transforms remains an intriguing and promising topic for further research.

\section{References}

1. Bernstein, S., Lanzani, L.: Szegö projections for Hardy spaces of monogenic functions and applications. Int. J. Math. Math. Sci. 29(10), 613-624 (2002)

2. Brackx, F., Bureš, J., De Schepper, H., Eelbode, D., Sommen, F., Souček, V.: Fundaments of Hermitean Clifford Analysis. Part I: Complex structure. Complex Anal. Oper. Theory 1(3), 341-365 (2007)

3. Brackx, F., Bureš, J., De Schepper, H., Eelbode, D., Sommen, F., Souček, V.: Fundaments of Hermitean Clifford Analysis. Part II: Splitting of h-monogenic equations. Complex Var. Elliptic Equ. 52(10-11), 1063-1079 (2007)

4. Brackx, F., De Knock, B., De Schepper, H.: A specific family of Clifford distributions. In: Son, L.H., Tutschke, W., Jain, S. (eds.) Methods of Complex and Clifford Analysis (Proceedings of ICAM, Hanoi, August 2004), pp. 215-228. SAS International Publication, Delhi (2004)

5. Brackx, F., De Knock, B., De Schepper, H.: Generalized Multi-Dimensional Hilbert transforms Involving Spherical Monogenics in the Framework of Clifford Analysis. In: Simos, T.E., Psihoyios, G., Tsitouras, Ch. (eds.) ICNAAM 2005, Official Conference of the European Society of Computational Methods in Sciences and Engineering, pp. 911-914. Wiley-VCH Verlag GmbH \& Co KGaA, Weinheim (2005)

6. Brackx, F., De Knock, B., De Schepper, H.: Multi-vector Spherical Monogenics, Spherical Means and Distributions in Clifford Analysis. Acta Math. Sin. (Engl. Ser.) 21(5), 1197-1208 (2005)

7. Brackx, F., De Knock, B., De Schepper, H.: A multidimensional Hilbert transform in anisotropic Clifford analysis. In: Gürlebeck, K., Könke, C. (eds.) Proceedings of the 17th International Conference on the Application of Computer Science and Mathematics in Architecture and Civil Engineering. Bauhaus Universität Weimar, Germany (12-14 July 2006) [cd-rom proceedings]

8. Brackx, F., De Knock, B., De Schepper, H.: Generalized Multidimensional Hilbert Transforms in Clifford analysis. Int. J. Math. Math. Sci. 2006, 1-19 (2006)

9. Brackx, F., De Knock, B., De Schepper, H.: On the Fourier Spectra of Distributions in Clifford Analysis. Chin. Ann. Math. Ser. B 27B(5), 495-506 (2006)

10. Brackx, F., De Knock, B., De Schepper, H.: On generalized Hilbert transforms and their interaction with the Radon transform in Clifford analysis. Math. Methods Appl. Sci. 30(9), 1071-1092 (2007)

11. Brackx, F., De Knock, B., De Schepper, H.: A metric dependent Hilbert transform in Clifford analysis. Bull. Belg. Math. Soc. Simon Stevin 14(3), 445-453 (2007)

12. Brackx, F., De Knock, B., De Schepper, H.: A matrix Hilbert transform in Hermitean Clifford analysis. J. Math. Anal. Appl. 344(2), 1068-1078 (15 August 2008)

13. Brackx, F., De Knock, B., De Schepper, H., Peña Peña, D., Sommen, F.: On Cauchy and Martinelli-Bochner integral formulae in Hermitean Clifford analysis, submitted.

14. Brackx, F., De Knock, B., De Schepper, H., Sommen, F.: Distributions in Clifford Analysis: an Overview. In: Eriksson, S.-L. (ed.) Clifford Analysis and Applications (Proceedings of the Summer School, Tampere, August 2004), pp. 59-73. Tampere University of Technology, Institute of Mathematics, Research Report 82 (2006)

15. Brackx, F., Delanghe, R., Sommen, F.: Clifford Analysis, Research Notes in Mathematics 76. Pitman Advanced Publishing Program, Boston - London - Melbourne (1982) 
16. Brackx, F., Delanghe, R., Sommen, F.: Spherical Means, Distributions and Convolution Operators in Clifford Analysis. Chin. Ann. Math. Ser. B 24B(2), 133-146 (2003)

17. Brackx, F., Delanghe, R., Sommen, F.: Spherical Means and Distributions in Clifford Analysis. In: Qian, T., Hempfling, T., McIntosch, A., Sommen, F. (eds.) Advances in Analysis and Geometry: New Developments Using Clifford Algebra, pp. 65-96. Trends in Mathematics, Birkhäuser, Basel (2004)

18. Brackx, F., De Schepper, H.: Hilbert-Dirac operators in Clifford analysis. Chin. Ann. Math. Ser. B 26B(1), 1-14 (2005)

19. Brackx, F., De Schepper, H., Sommen, F.: The Hermitian Clifford analysis toolbox, to appear in Adv. Appl. Clifford Alg. (online first).

20. Brackx, F., De Schepper, N., Sommen, F.: The Clifford-Fourier Transform. J. Fourier Anal. Appl. 11(6), 669-681 (2005)

21. Brackx, F., De Schepper, N., Sommen, F.: The two-dimensional Clifford-Fourier Transform. J. Mat: Imaging Vis. 26(1-2), 5-18 (2006)

22. Brackx, F., De Schepper, N., Sommen, F.: Metric dependent Clifford analysis with applications to wavelet analysis. In: Alpay, D. (ed.) Wavelets, multiscale systems and hypercomplex analysis, Operator Theory: Advances and Applications 167, pp. 17-67. Birkhäuser Verlag, Basel (2006)

23. Brackx, F., De Schepper, N., Sommen, F.: The Fourier Transform in Clifford Analysis. Advances in Imaging and Electron Physics 156(6), 55-201 (2008)

24. Bülow, T., Sommer, G.: The hypercomplex signal - a novel approach to the multidimensional signal. IEEE Trans. Signal Process. 49(11), 2844-2852 (2001)

25. Calderbank, D.: Clifford analysis for Dirac operators on manifolds with boundary. MaxPlanck-Institut für Mathematik, Bonn (1996)

26. Cnops, J.: An introduction to Dirac operators on manifolds, Progress in Mathematical Physics 24. Birkhäuser, Boston (2002)

27. Delanghe, R.: Clifford analysis: history and perspective. Comput. Methods Funct. Theory $\mathbf{1}(1), 107-153(2001)$

28. Delanghe, R.: Some Remarks on the Principal Value Kernel in $\mathbb{R}^{m}$. Complex Var. Theory Appl. 47(8), 653-662 (2002)

29. Delanghe, R.: On some properties of the Hilbert transform in Euclidean space. Bull. Belg. Math. Soc. Simon Stevin 11, 163-180 (2004)

30. Delanghe, R., Sommen, F., Souček, V.: Clifford Algebra and Spinor-Valued Functions, A Function Theory for the Dirac Operator, Mathematics and its Applications 53. Kluwer Academic Publishers, Dordrecht (1992)

31. De Schepper, N.: Multi-dimensional Continuous Wavelet Transforms and Generalized Fourier Transforms in Clifford Analysis, PhD-thesis. Ghent University, Ghent (2006)

32. Ebling, J., Scheuermann, G.: Clifford Convolution and Pattern Matching on Vector Fields. In: Proceedings of IEEE Visualization '03, pp. 193-200. Computer Society, Los Alamos, CA, USA (2003)

33. Ebling, J., Scheuermann, G.: Clifford Fourier Transform on Vector Fields. IEEE Trans. Vis. Comput. Graph. 11(4), 469-479 (2005)

34. M. Felsberg, Low-Level Image Processing with the Structure Multivector, PhD-thesis, Christian-Albrechts-Universität, Kiel, 2002.

35. Gilbert, J.E., Murray, M.A.M.: Clifford Algebra and Dirac Operators in Harmonic Analysis, Cambridge Studies in Advanced Mathematics 26. Cambridge University Press, Cambridge (1991)

36. Horváth, J.: Sur les fonctions conjuguées à plusieurs variables (French). Nederl. Akad. Wetensch. Proc. Ser. A 56 = Indagationes Mathematicae 15, 17-29 (1953)

37. J. Horváth, Singular Integral Operators and Spherical Harmonics, Trans. Amer. Math. Soc., 82, 1950, 52-63.

38. Li, C., McIntosh, A., Qian T.: Clifford algebras, Fourier transforms, and singular convolution operators on Lipschitz surfaces, Revista Mathematica Iberoamericana 10, 665-721 (1994)

39. Mawardi, B., Hitzer, E.: Clifford Fourier Transformation and Uncertainty Principle for the Clifford Geometric Algebra $\mathrm{Cl}_{3,0}$. Adv. Appl. Clifford Alg. 16(1), 41-61 (2006) 
40. McIntosh, A.: Clifford algebras, Fourier theory, singular integrals and harmonic functions on Lipschitz domains. In: J. Ryan (ed.) Clifford Algebras in Analysis and Related Topics (Fayetteville, 1993), Studies in Advanced Mathematics, pp. 33-87. CRC Press, Boca Raton (1996)

41. A.D. Poularikas (ed.), The transforms and applications handbook, CRC Press, Boca Raton, 1996.

42. Rocha-Chavez, R., Shapiro, M., Sommen, F.: Integral theorems for functions and differential forms in $\mathbb{C}_{m}$, Research Notes in Mathematics 428. Chapman \& Hall/ CRC, New York (2002)

43. Ryan, J.: Clifford Analysis. In: Ablamowicz R., Sobczyk G. (eds.), Lectures on Clifford (Geometric) Algebras and Applications, Birkhäuser, Boston (2004), 53-89

44. Ryan, J.: Complexified Clifford analysis. Complex Var. Theory Appl. 1(1), 119-149 $(1982 / 83)$

45. Sabadini, I., Sommen, F.: Hermitian Clifford analysis and resolutions. Math. Methods Appl. Sci. 25(16-18), 1395-1413 (2002)

46. Sommen, F.: Some connections between Clifford analysis and complex analysis. Complex Var. Theory Appl. 1(1), 97-118, (1982)

47. Sommen, F.: Hypercomplex Fourier and Laplace transforms II. Complex Var. Theory Appl. 1(2-3), 209-238 (1982/83) 